\title{
Site Selectivity for CO Adsorption and Stripping on Stepped and Kinked Platinum Surfaces in Alkaline Medium
}

Manuel J. S. Farias, Enrique Herrero, Juan M. Feliu* Instituto de Electroquímica, Universidad de Alicante Ap. 99, E-03080, Alicante, Spain.

\begin{abstract}
This work reports experimental evidences that the adsorption of $\mathrm{CO}$ on Pt surfaces composed by (111) terraces and steps and/or kinks in alkaline media occurs faster on sites with (100) symmetry, followed by (110) sites. On the other hand (111) terrace have the lowest adsorption rate. $\mathrm{CO}$ electrooxidation demonstrates the existence of a close relationship between the preferential site $\mathrm{CO}$ occupancy and the peak multiplicity observed in CO stripping voltammetry. For the stepped $\operatorname{Pt}(554)$ and $\operatorname{Pt}(544)$ and kinked $\operatorname{Pt}(875)$ single crystal surfaces, the CO stripping process takes place at high potentials ( 0.80 V vs RHE) when only the (110) or (100) step sites are blocked by CO. However, when the terrace sites with (111) symmetry are fully occupied, new CO stripping peaks appear at lower potentials $(<0.68 \mathrm{~V} v s$. RHE). For all surfaces, it is observed that the first released sites after partial CO oxidation are (111) terrace sites, followed by the step (110) sites and sites with (100) symmetry. The results of partial CO oxidation suggest that the diffusion of adsorbed CO from sites with (110) or (100) symmetry towards unoccupied (111) terrace sites is negligible. However, $\mathrm{CO}$ diffusion from terrace sites to step or kink sites cannot be discarded during the growth of the adsorbed CO adlayer, because of the preferential site occupancy for these latter sites. Due to the fact that CO oxidation process on stepped Pt surfaces in alkaline media occur at different potentials on different sites, the activation energy for $\mathrm{CO}$ oxidation on the different sites have been
\end{abstract}


estimated. The results for full $\mathrm{CO}$ coverage and $\mathrm{CO}$ decorated stepped surfaces are in good agreement, indicating that the oxidation of $\mathrm{CO}$ on the different sites is not coupled. In absence of $\mathrm{CO}$ on terrace sites, in situ FTIR spectroscopy shows that CO molecules on the (110) step sites are essentially linearly bonded, while on the (100) step sites both linearly and bridge bonded $\mathrm{CO}$ are observed. The comparison of these spectra with those obtained when a full coverage is attained shows that the band frequencies for $\mathrm{CO}$ on step sites are highly coupled with those on the terrace sites.

Keywords: Electrocatalysis; carbon monoxide; platinum single crystals; alkaline medium.

* Corresponding authors: E-mail address: juan.feliu@ua.es (J. M. Feliu). Phone: +34 965909301 


\section{Introduction}

In recent years, several systematic studies have been published concerning the influence of controlled amounts of surface defects in $\mathrm{CO}$ electrocatalysis. ${ }^{1-4}$ On this particular issue, step sites on (111) terraces have been recognized to act preferentially as nucleation center for oxygen-containing species. ${ }^{5}$ This statement comes from the experimental fact that the $\mathrm{CO}$ electrocatalysis is always enhanced when crystalline or randomly defect sites were introduced onto a flat (111) surface. Based on this fact, the diffusion of $\mathrm{CO}$ on the terraces has been assumed to be faster than the rate of $\mathrm{CO}$ oxidation reaction itself, so that $\mathrm{CO}$ oxidation reaction only occurs at step sites. ${ }^{1}$ This interpretation is supported by the measurements carried out in UHV environments, in which $\mathrm{CO}$ adsorption starts on the step sites and then continues on the terrace sites ${ }^{6-10}$ For electrochemical interfaces, very few studies describe the influence of crystalline defects in the adsorption behavior of CO on stepped surfaces. ${ }^{11-12}$ Additionally, it has been found that the preferential step site occupancy acidic media may depend on the step symmetry. ${ }^{13}$ It has been observed for $\operatorname{Pt}(\mathrm{s})-[n(111) \times(100)]^{12}$ surfaces but not for the $\operatorname{Pt}(\mathrm{s})-[n(111) \times(110)]$ surfaces, probably related to different experimental conditions. ${ }^{14}$ This fact makes difficult the interpretation of the data based on the mobility/dynamics of CO layers at the electrochemical interface, and therefore complicates the proposal of a realistic kinetic model for this reaction. ${ }^{15-16}$

The lack of reliable data on the preferential site occupancy is more evident in alkaline media, especially for single crystal electrodes. In some of the few studies regarding this issue, Garcia et al. ${ }^{17-20}$ have suggested a site reactivity order for CO electrooxidation on stepped platinum surfaces in alkaline media. The least active site for CO oxidation would be the (111) terrace site, while the (110) step sites would promote the electrooxidation of $\mathrm{CO}$ at lower potential values than the (100) step sites. The 
general framework proposed by Garcia et al. is similar to that reported for the $\mathrm{CO}$ oxidation reaction in acidic media, in which the role of crystalline step sites is to activate oxygen-containing species, which finally react with $\mathrm{CO}$, with a major difference: the much slower CO diffusion in alkaline media. ${ }^{21}$ From the results reported in the literature, it is very clear that the reactivity of stepped platinum surfaces toward $\mathrm{CO}$ electrooxidation depends on the local symmetry of the surface sites. However, it is not clear whether the defects promote the activation of oxygen-containing species in $\mathrm{CO}$ oxidation catalysis especially in alkaline medium.

For a given platinum surface, the electrocatalytic activity for the $\mathrm{CO}$ oxidation reaction in alkaline media is higher than that measured in acidic media. Although the origin of such enhancement is not known, some authors have ascribed it to a higher affinity for the adsorption of $\mathrm{OH}$ at the defect sites. ${ }^{22-23}$ On the other hand, it has been proposed that the $\mathrm{CO}$ diffusion coefficient on platinum surfaces in alkaline environments $\left(\sim 2.7 \times 10^{-16} \mathrm{~cm}^{2} \mathrm{~s}^{-1}\right)$ is significantly smaller than measured in acidic medium. $^{21}$

A very interesting feature of a full $\mathrm{CO}$ monolayer oxidation on Pt stepped surfaces in alkaline media is that different processes, which depend on the sites present on the surface, can be observed at different electrode potentials in a $\mathrm{CO}$ stripping experiment. Thus, properties such as the potential dependence of the band frequency and the activation energies can be studied separately for a given type site on different stepped and kinked surfaces. In acidic media, Kim and Korzeniewski ${ }^{12}$ using low CO coverages on the $\operatorname{Pt}(\mathrm{s})-[n(111) \times(100)]$ surfaces have reported separated band frequencies for adsorbed $\mathrm{CO}$ at differents structural sites. The individual $\mathrm{CO}$ band frequencies are highly affected by the dipole-dipole coupling, ${ }^{24-26}$ so that it is very difficult to study the band behavior for $\mathrm{CO}$ on step sites without the coupling interference of $\mathrm{CO}$ on terrace 
sites. However, as will be shown, this limitation can be overcome by using a careful CO stripping procedure. Also, activation energy values have been estimated in acidic media for the Pt basal planes at constant dosing potentials ${ }^{27-28}$ although Rincon et al. ${ }^{29}$, working with a polycrystalline platinum wire, showed that the activation energy for $\mathrm{CO}$ stripping is highly dependent on the $\mathrm{CO}$ adsorption potential.

Direct macroscopic evidences on the influence of crystalline defects in both $\mathrm{CO}$ adsorption and their subsequent electrooxidation can be obtained from the hydrogen adsorption signal on the surface during $\mathrm{CO}$ dosing and $\mathrm{CO}$ stripping experiments. This analysis is possible because adsorption processes on platinum single crystal electrodes are very sensitive to the local environment. ${ }^{30}$ Thus, the evolution of the voltammogram can be used to analyze these processes on the surface, because the interactions of molecules with the surface produce different modifications depending on whether the interaction occurs with (111), (110) or (100) sites. ${ }^{14,31}$

This paper aims to characterize the processes of $\mathrm{CO}$ filling/release on both stepped and kinked platinum surfaces in alkaline media in slow dosing and partial $\mathrm{CO}$ stripping experiments. The analysis of consecutive experiments allows pointing out the origin of peak multiplicity in CO stripping on those surfaces. An experimental sequence of site release after partial $\mathrm{CO}$ stripping from a fully $\mathrm{CO}$ covered surface will be described at different sweep rate, characterizing these processes with their corresponding Tafel plots. We also report on the local activation energies for $\mathrm{CO}$ electrooxidation on the different sites: (110) and (100) symmetry sites and terrace sites with (111) symmetry with medium length (9-5 atomic rows). Using in situ FTIR spectroscopic data, the exact CO coordination on both step sites in absence of $\mathrm{CO}$ on terrace sites has been identified. 


\section{Experimental}

Platinum electrodes with (554), (544) and (875) orientations were prepared from single crystal beads according to procedure described in reference. ${ }^{32}$ The $\operatorname{Pt}(554)$ and Pt(544) electrode are stepped surfaces containing 9 atom-wide (111) terraces separated by monoatomic steps with (110) and (100) symmetry, respectively. They can be described as:

$$
\begin{aligned}
& \operatorname{Pt}(554) \equiv \operatorname{Pt}(s)-[9(111) \times(110)] \\
& \operatorname{Pt}(544) \equiv \operatorname{Pt}(s)-[9(111) \times(100)]
\end{aligned}
$$

On the other hand, the equivalent notation for the $\mathrm{Pt}(875)$ kink surface is:

$$
\operatorname{Pt}(875) \equiv \operatorname{Pt}(s)-[5(111) \times 3(110) \times(100)]
$$

which indicates that this surface contains 5 atom-wide (111) terraces separated by monoatomic steps containing (110) sites symmetry broken by (100) sites, which define kinks nominally every 3 step sites.

The electrodes were flame annealed and cooled down in a $\mathrm{H}_{2}+$ Ar atmosphere, as usual. This procedure has been revealed useful to prepare stepped surfaces whose topography agrees with the nominal one, ${ }^{33}$ although no data are available for kinked surfaces. A platinized platinum wire was used as a counter electrode, and a reversible hydrogen electrode (RHE) was employed as reference electrode. Voltammetric curves measured in alkaline media were used to estimate the two-dimensional order of the surface, as well as solution cleanliness, which was also checked with a $\operatorname{Pt}(111)$ electrode. All electrochemical and spectroelectrochemical experiments were carried out using a waveform generator (EG\&G PARC 175) together with a potentiostat (Amel 551) and a digital recorder (eDAC ED 401). The temperature control was achieved by a digital temperature controller (PolyScience ${ }^{\circledR}$ ). 
In situ FTIR spectra were obtained using a Nicolet (Model 8700) spectrometer, equipped with an MCT (Mercury-Cadmium-Telluride) detector. The different spectra (obtained from the average of 100 interferograms using a resolution of $8 \mathrm{~cm}^{-1}$ ) were collected at potential steps from $0.05 \mathrm{~V}$ to $0.30 \mathrm{~V} v s$. RHE (onset of CO oxidation), at intervals of $25 \mathrm{mV}$. After that, the electrode potential was stepped to $0.90 \mathrm{~V}$ to collect the reference spectrum. For all experiments, $p$-polarized radiation was employed, which allows detecting active species both on the electrode surface and the electrolyte solution. ${ }^{34}$ Spectra are presented in absorbance units $\left(A=-\log \left(R / R_{0}\right)\right)$ in which $R$ and $R_{o}$ refer to the reflectance spectra at the sampling and reference potential $(0.90 \mathrm{~V})$, respectively.

Experiments were carried out in $0.1 \mathrm{~mol} \mathrm{~L}^{-1} \mathrm{NaOH}$ (Merck KGaA, $99.99 \%$ ) prepared in ultrapure water (Millipore Milli-Q 18.2 $\mathrm{M} \Omega \mathrm{cm}$ ). The CO (Alpha Gas, $99.997 \%$ ) sub-monolayer was deposited under potentiostatic conditions by using a low gas flow in the electrochemical cell atmosphere.

\section{Results}

\subsection{Cyclic voltammograms in electrolyte solution}

The voltammetric profiles of the single crystal electrodes used in this work in 0.1 $\mathrm{M} \mathrm{NaOH}$ are shown in Figure 1. The stability of these profiles shows that the electrolyte solution is free from impurities, and the current distribution indicates that the surfaces are very well ordered. Additionally, a hard sphere model of the surfaces is also included in this figure for clarity. 


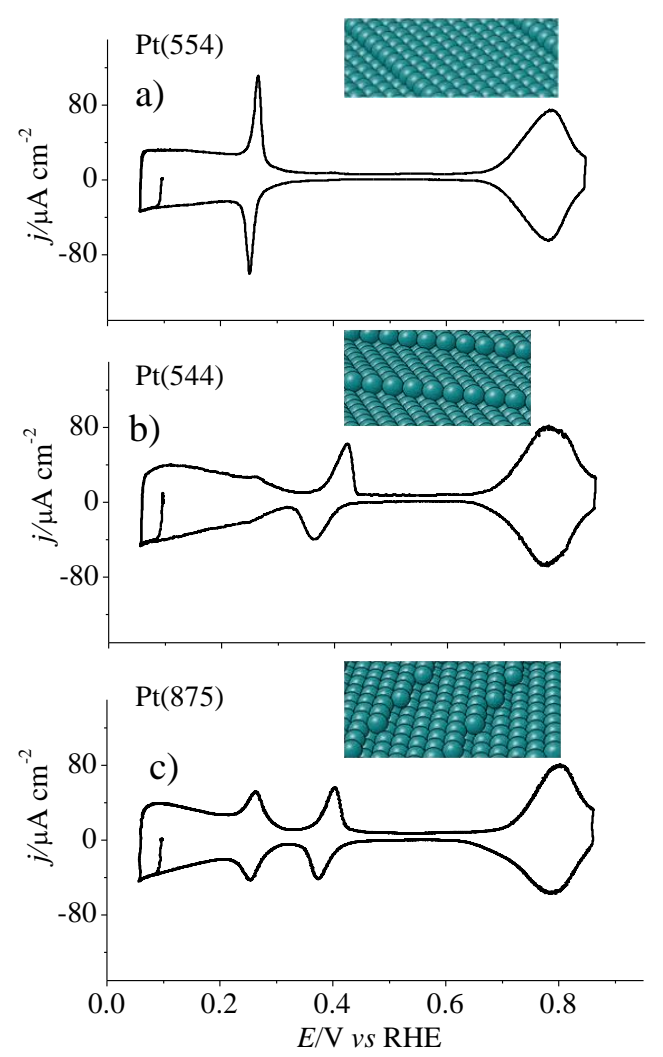

Figure 1. Cyclic voltammograms and atomic model of single crystals. Sweep rate $0.05 \mathrm{~V} \mathrm{~s}^{-1}$.

The couple of peaks at around 0.26 V for the stepped $\mathrm{Pt}(554)$ surface (Figure 1a) is characteristic of the so-called hydrogen adsorption/desorption at (110) step sites. In the same way, for $\operatorname{Pt}(544)$ surface, the less reversible couple of peaks at 0.42 (positivegoing sweep) and $0.36 \mathrm{~V}$ (negative-going sweep) is characteristic of this process on the (100) step. It should be mentioned that the $\operatorname{Pt}(544)$ electrode used in this work has a small amount of (110) defects as revealed by the small shoulder observed at $0.26 \mathrm{~V}$. For the kinked $\mathrm{Pt}(875)$ surface, the corresponding pairs of peaks at $0.26 \mathrm{~V}$ and $0.40 \mathrm{~V}$ can be relate to hydrogen adsorption/desorption on (110) step and (100) kink sites, respectively. It is worth mentioning that the reversibility of this latter couple of peaks is significantly higher than that observed on the corresponding stepped surface with (100) step sites $\left(\Delta E_{\mathrm{p}} \approx 60 \mathrm{mV}\right)$. Regarding the nature of these peaks associated with the 
step/kink sites, it has been proposed that they are due to the competitive adsorption of hydrogen and probably $\mathrm{OH} / \mathrm{O}$ species. ${ }^{35}$

\subsection{Potentiostatic CO dosing}

Figure 2 shows the voltammetric profiles for successive $\mathrm{CO}$ sub-monolayers obtained by slow $\mathrm{CO}$ dosing at $0.1 \mathrm{~V}$. The growth of the $\mathrm{CO}$ sub-monolayer was controlled by maintaining a very low $\mathrm{CO}$ gas flow through the cell atmosphere (around $10 \%$ of the argon flow through the cell atmosphere) for different dosing times. After some particular dosing times, control cyclic voltammograms were recorded at $0.20 \mathrm{~V} \mathrm{~s}^{-}$ 1. Using this relatively fast scan rate and a low CO gas flow, it is possible to minimize the interferences of $\mathrm{CO}$ from solution while recording the cyclic voltammogram. Each recording cycle lasts approximately 3 seconds only, and thus, the $\mathrm{CO}$ coverage change in each cycle can be considered negligible. This experimental procedure was previously

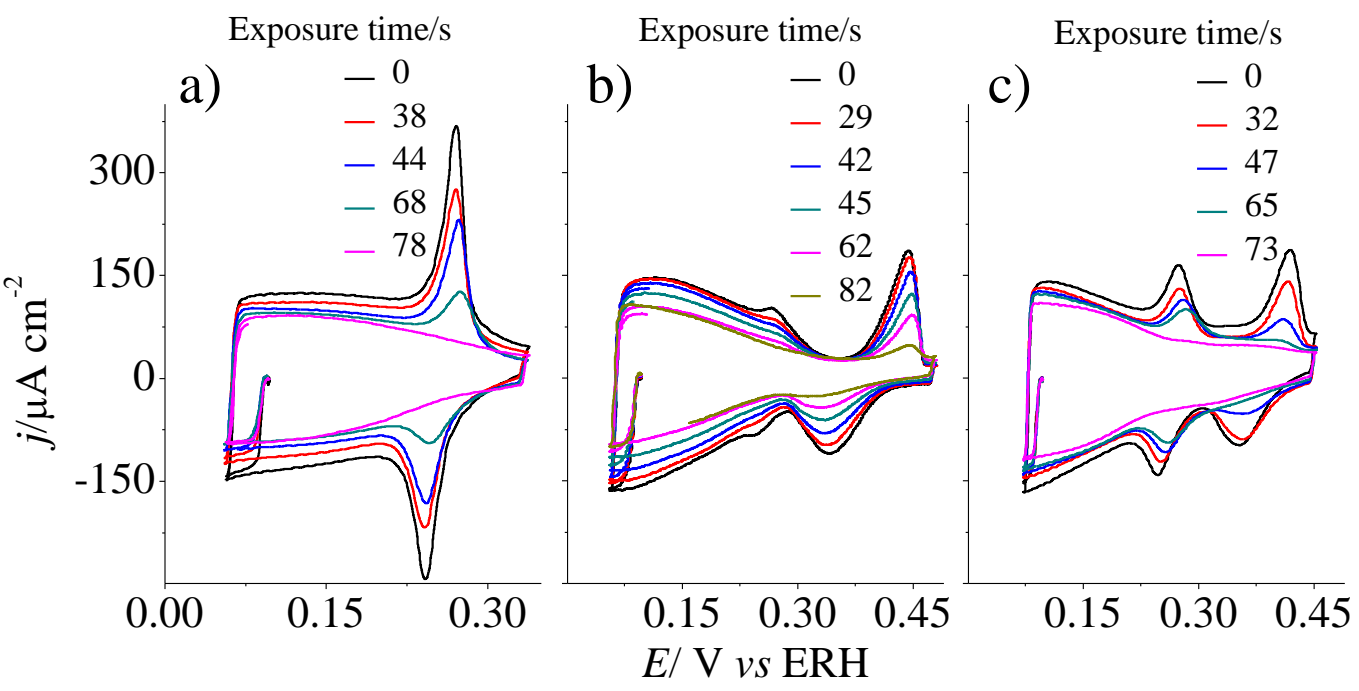

Figure 2. Cyclic voltammograms between $0.05 \mathrm{~V}$ and $0.40 \mathrm{~V}$ for: a) $\mathrm{Pt}(554)$; and b) $\operatorname{Pt}(544)$; and c) Pt(875) electrodes. Data recorded after the potentiostatic growth of the CO submonolayer at $0.10 \mathrm{~V}$. The different exposure times correspond to different degrees of $\mathrm{CO}$ coverage. Sweep rate: $0.20 \mathrm{~V} \mathrm{~s}^{-1}$ 
checked and reported in acidic medium. ${ }^{14}$

The comparison of the voltammograms in Figure 2a-b shows that site blocking occurs at a higher rate for (110) and (100) step sites than that measured for (111) terrace sites, since the peak associated to the hydrogen adsorption on these peaks disappears well before the terrace sites have been completely blocked. In order to quantitatively asses the site blocking rate, the charge for these peaks and for hydrogen adsorption on the terrace is calculated and plotted in Figure 3 vs. the $\mathrm{CO}$ dosing time. Because of the experimental difficulties to measure the contribution on the hydrogen region on (111) terraces for the $\operatorname{Pt}(875)$ electrode, the total charge density minus that due to step/kink sites $\left[\sigma_{\text {total }}-\sigma_{(110)}-\sigma_{(100)}\right]$ is shown. If CO adsorption were insensitive to the adsorption site, it would be expected that the complete blockage of the different sites on the surface took place at the same dosing time. However, the complete blockage of the step/kink sites occurs well before that of the terrace. In fact, the rate of step site blocking becomes even faster when there is a partial CO coverage on the steps, as revealed from the fast diminution of these signals after ca. $40 \mathrm{~s}$ dosing time. This dosing time corresponds to a total blockage of the hydrogen sites of ca $16 \%$. Likewise, the hydrogen site blocking

a)

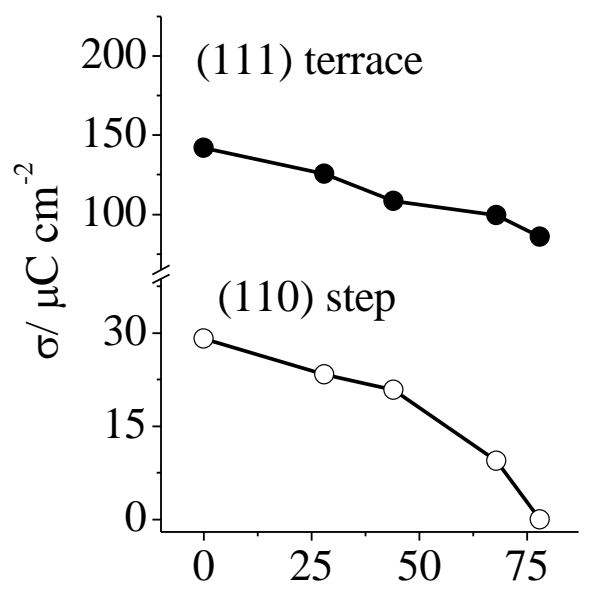

b)

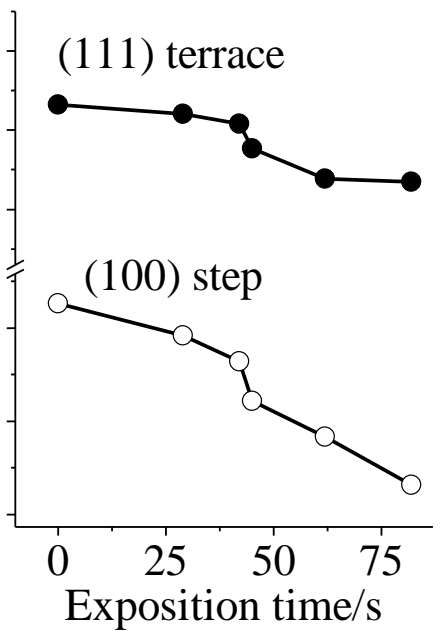

c)

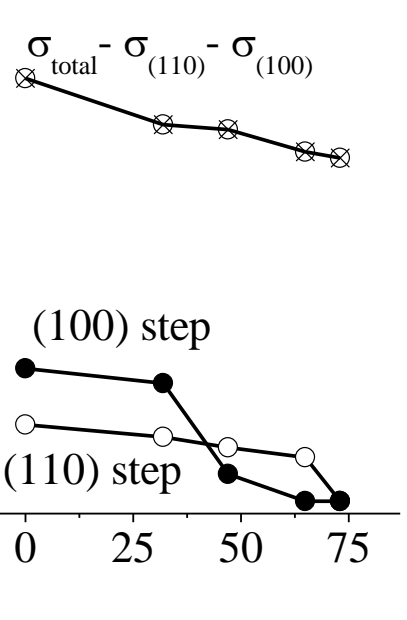

Figure 3. Charge under the voltammetric peaks $\left(\sigma_{\text {step }}\right.$ and $\left.\sigma_{\text {terrace }}\right) v s$. time: a) $\operatorname{Pt}(554)$; b) $\operatorname{Pt}(544)$ and c) Pt(875) electrodes. Data calculated from Figure 2. 
during $\mathrm{CO}$ sub-monolayer growth onto a kinked $\mathrm{Pt}(875)$ surface in Figure $2 \mathrm{c}$ occurs with higher rate for sites with (100) symmetry as compared to those having (110) symmetry. Again, the last type of sites to be blocked is that corresponding to (111) terraces, as observed for the stepped surfaces.

The above results show that the pattern of $\mathrm{CO}$ site occupancy onto stepped and kinked platinum surfaces are quite similar. Furthermore, the pattern of site blocking for all surfaces is independent of whether the $\mathrm{CO}$ adsorption is carried out under potentiostatic or potentiodynamic (not shown) conditions. This last result makes reliable the surface blockage study described above.

\subsection{Effect of $C O$ sub-monolayer growth on the $C O$ stripping voltammetry}

It is already known that $\mathrm{CO}$ stripping voltammetry for stepped platinum surfaces in alkaline media exhibits peak multiplicity. ${ }^{17,} 21$ In this section, we attempt to investigate the conditions required to give rise to the multiple peaks in $\mathrm{CO}$ stripping voltammetry. The strategy is to compare site blockage in the hydrogen adsorption region with peak position for CO stripping. Figure 4 shows that the voltammetric profile of $\mathrm{CO}$ stripping is very dependent on the $\mathrm{CO}$ coverages obtained after controlled growth of the $\mathrm{CO}$ sub-monolayer. These experiments were carried out as follows: a weak $\mathrm{CO}$ flow of $\mathrm{CO}$ was introduced in the cell atmosphere near the electrode meniscus (as done in the experiments reported in the Figure 2), and the surface blockage after some dosing time was examined. After the desired coverage value was reached, the $\mathrm{CO}$ submonolayer was stripped in a single scan up to $0.85 \mathrm{~V}$ at $0.20 \mathrm{~V} \mathrm{~s}^{-1}$. As before, this relatively fast scan rate was used to avoid interferences from $\mathrm{CO}$ in the solution. The measured voltammetric profile for $\mathrm{CO}$ stripping does not differ essentially from that measured in a solution free of $\mathrm{CO}$. 


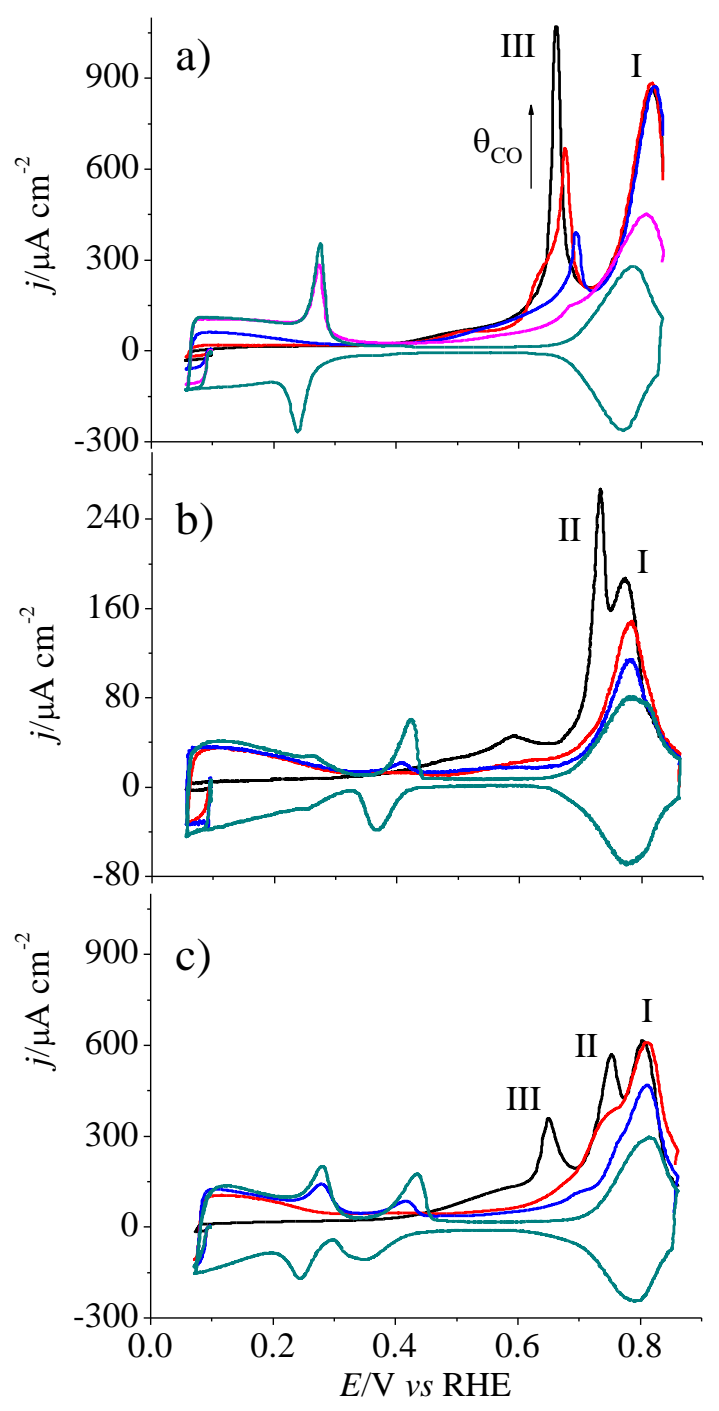

Figure 4. CO stripping voltammetry after partial potentiostatic growth of the $\mathrm{CO}$ submonolayer at $0.10 \mathrm{~V}$ for a) $\mathrm{Pt}(554)$, b) $\mathrm{Pt}(544)$,and c) $\mathrm{Pt}(875)$ electrodes. In all cases, the dark cyan line is blank voltammetry,the black line corresponds to a full $\mathrm{CO}$ coverage, and the other lines are for intermediate CO coverages; Data a) and c) were recorded at $0.20 \mathrm{Vs}^{-1}$ and b) was recorded at $0.05 \mathrm{Vs}^{-1}$.

For the $\mathrm{Pt}(554)$ electrode (Figure 4a), it can be observed that above a certain $\mathrm{CO}$ coverage (blue, red and black lines), the CO stripping voltammetry displays two peaks, denoted by I and III, at $0.81 \mathrm{~V}$ and $0.68 \mathrm{~V}$, respectively. Apart from scan rate effects, peak I always develops in a potential region that coincides with that of the oxide/hydroxide peak $(\sim 0.78 \mathrm{~V})$. On the other hand, the peak III is located in the socalled double layer region. After reaching the maximum charge for process I, the process III gradually increases with increasing $\mathrm{CO}$ coverage. By comparing the 
voltammogram in the lower potential region with that in the $\mathrm{CO}$ stripping region, it is clear that the current density for process $\mathrm{I}$ is maximum when the blockage of the hydrogen adsorption at (110) step sites is complete. The process III appears only when the hydrogen adsorption on (111) terrace sites is almost fully blocked. Furthermore, there is a previous process especially for full $\mathrm{CO}$ coverage, which is usually named as the pre-oxidation wave. Previous results have shown that this prewave is not associated with defect or kinks in the electrode surface. ${ }^{21}$

A very similar behavior is observed for $\operatorname{Pt}(544)$ electrode (Figure $4 b$ ). For a saturated CO layer two peaks are observed in the stripping voltammogram at $0.05 \mathrm{~V} \mathrm{~s}^{-1}$ : peak I at $0.77 \mathrm{~V}$ and peak II at $0.73 \mathrm{~V}$. Due to the possible overlapping of both peaks at a scan rate of $0.20 \mathrm{~V} \mathrm{~s}^{-1}$, this experiment was exceptionally recorded at $0.05 \mathrm{~V} \mathrm{~s}^{-1}$. However, it should be mentioned that the voltammograms of $\mathrm{CO}$ stripping at submonolayer coverage do not differ from those recorded in $\mathrm{CO}$ free solutions. Initially, only peak I at $0.77 \mathrm{~V}$ is present on the surface. As the $\mathrm{CO}$ coverage increases, this peak progressively grows. Only when all the terrace sites are fully blocked, peak II appears at $0.73 \mathrm{~V}$. Moreover, this particular surface also contains a minor amount of defect sites with (110) symmetry, that behave similarly to that described for $\operatorname{Pt}(554)$, see arrows in Figure 4b. In this respect, a small peak III is also observed at full surface blockage, overlapping the prewave.

The kinked $\mathrm{Pt}(875)$ surface (Figure 4c) contains all processes I, II and III. Again the last two processes only appear when the (111) terrace sites become almost fully blocked. It is interesting to remark that peak I appears at $0.80 \mathrm{~V}$, e.g. an intermediate value as compared with the stepped surfaces. Also, peak II appears at $0.75 \mathrm{~V}$ and peak III at $0.64 \mathrm{~V}$ at this kinked $\operatorname{Pt}(875)$ surface. We have also observed that the pre-wave is always observed when stripping a saturated $\mathrm{CO}$ layer, regardless the presence of traces 
of CO in solution. To sum up, peak I appears for all the CO coverages, whereas peaks II and III are only observed when all terrace sites have been blocked.

\subsection{Identification of preferential site release by partial $\mathrm{CO}$ adlayer stripping}

Figure 5 shows various partial CO stripping experiments. After the growth of a fully blocked $\mathrm{CO}$ adlayer on each single crystal surface by $\mathrm{CO}$ dosing at $0.10 \mathrm{~V}$, the electrolyte was degassed for 20 minutes. Next, the $\mathrm{CO}$ adlayer was partially stripped by cycling the electrode potential up to a controlled upper potential limit. As a consequence, hydrogen adsorption on the (111) terrace sites is recovered. For the $\operatorname{Pt}(554)$ and $\operatorname{Pt}(875)$ single crystal electrodes, the electrode potential window was extended up to $0.70 \mathrm{~V}$ and $0.65 \mathrm{~V}$, respectively, in a first conditioning cycle $\left(\mathrm{C}_{1}\right.$, not shown). To achieve the same goal, two cycles up to $0.74 \mathrm{~V}$ were required for $\operatorname{Pt}(544)$ electrode. Once the conditioning cycle(s) were completed, the electrode potential was returned to $0.10 \mathrm{~V}$ and the potential window was gradually increased for each subsequent cycle $\left(\mathrm{C}_{2}, \mathrm{C}_{3}\right.$, etc $)$ in the potential range in which peak I develops. In these cycles, only a small fraction of $\mathrm{CO}$ was oxidized in each cycle. 


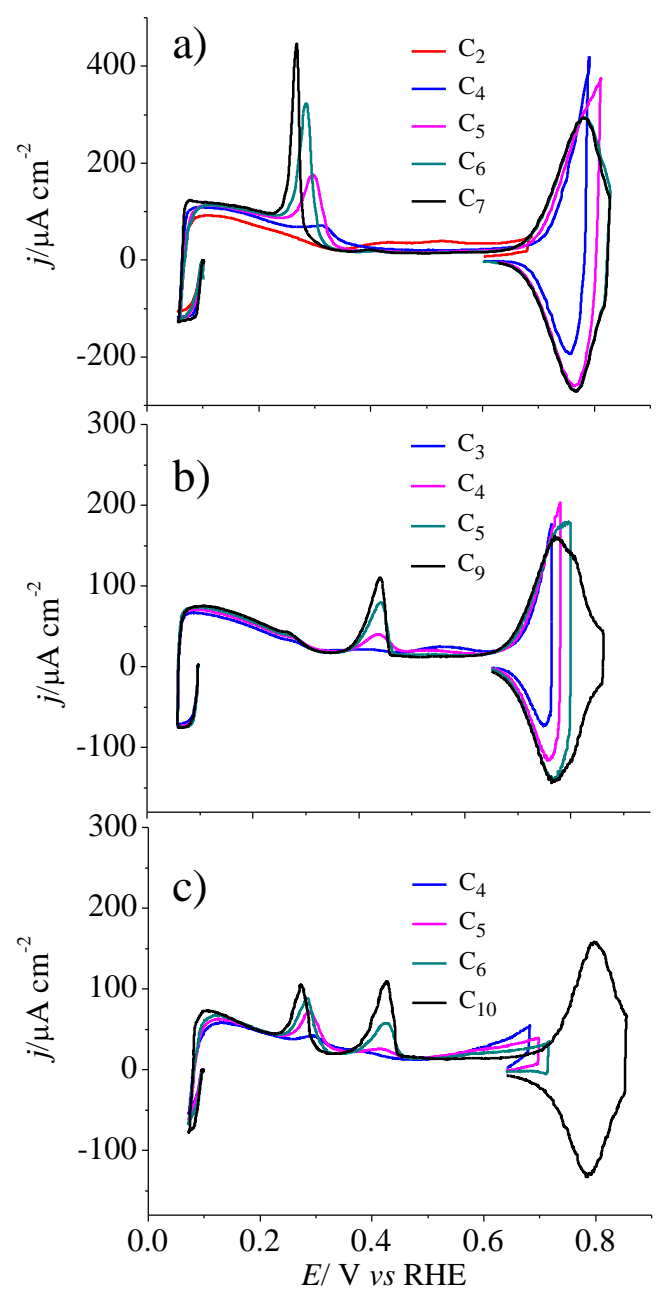

Figure 5.Partial $\mathrm{CO}$ oxidation experiments by selecting a low upper potential limit for a) $\mathrm{Pt}(554)$ and b) $\mathrm{Pt}(544)$ and c) $\mathrm{Pt}(875)$ single crystal electrodes. The data in a) was recorded at $0.20 \mathrm{Vs}^{-1}$; and the data in b) and c) were recorded at $0.10 \mathrm{Vs}^{-1}$. Only a few cycles are shown.

For the CO sub-monolayer adsorbed on the $\mathrm{Pt}(554)$ electrode after removal of process III (Figure 5a), the electrooxidation involves the gradual release of the sites available for hydrogen adsorption on the (110) step symmetry. Thus, process III should be associated with $\mathrm{CO}$ oxidation on some (111) terrace sites. It should be highlighted that the peak position for the adsorption on the (110) steps sites shifts towards lower potential values as $\mathrm{CO}$ is stripped from the surface, until it reaches the typical value when the CO layer has been completely stripped. This fact reflects different adsorption energies on the (110) sites depending on the local CO coverages. Such potential shift 
was not observed when similar CO coverages were attained by slow $\mathrm{CO}$ dosing (Figure 2), as slow CO dosing does not change the position of the peak associated with the (110) steps. These differences clearly indicate that the CO sub-monolayer with partial CO coverages on the steps depends on the procedure used to prepare the adlayer.

A similar situation is found for the $\operatorname{Pt}(544)$ electrode (Figure 5b): the oxidation of the remaining $\mathrm{CO}$ after complete removal of peak II involves the release of the step sites. For the kinked surface, the complete removal of the $\mathrm{CO}$ sub-monolayer in successive cycles allow determining which sites, (100) kinks or (110) step sites, are released faster. As can be seen, (100) sites are recovered more slowly than those having (110) symmetry. In all cases, the peak potential for the (100) adsorption state is less affected by the partial CO coverage on the step than the (110) step site.

\subsection{Influence of sweep rate on the peak potential for electrooxidation of bonded $\mathrm{CO}$ on terrace and step sites}

Figures 6 and 7 show the effect of the scan rate in the stripping voltammograms for $\mathrm{CO}$ oxidation at full (Figures $6 \mathrm{a}$ and $7 \mathrm{a}$ ) and partial coverages obtained after the elimination of peaks III and II (Figures $6 \mathrm{~b}$ and $7 \mathrm{~b}$ ) on the $\operatorname{Pt}(554)$ and $\operatorname{Pt}(544)$ surfaces. For a better comparison, capacitance curves (current densities divided by sweep rate) are shown. From the representation of the peak potential for each process, $E_{\mathrm{p}}$, versus the logarithm of the scan rate, the apparent Tafel slope of the different processes can be obtained. ${ }^{36}$ In all cases, CO oxidation in peaks II and III shows a higher shift on potential, as already observed by Garcia et al. ${ }^{17}$ and Herrero et al. ${ }^{21}$. For $\operatorname{Pt}(554)$ electrode at full coverage, the measured Tafel slope values are $42 \pm 3 \mathrm{mV}$ and $70 \pm 3$ $\mathrm{mV}$ for peak I and III respectively, whereas a value of $39 \pm 3 \mathrm{mV}$, for peak I is obtained from Figure $6 \mathrm{~b}$. These values are similar to those previously measured for the same 
electrode. Garcia et al. ${ }^{17}$ obtained value of $\mathrm{d} E / \mathrm{d}(\log v)$ around $36 \mathrm{mV}$ and $92 \mathrm{mV}$ for peaks I and III respectively, wherea Tafel slope values from chronoamperometric

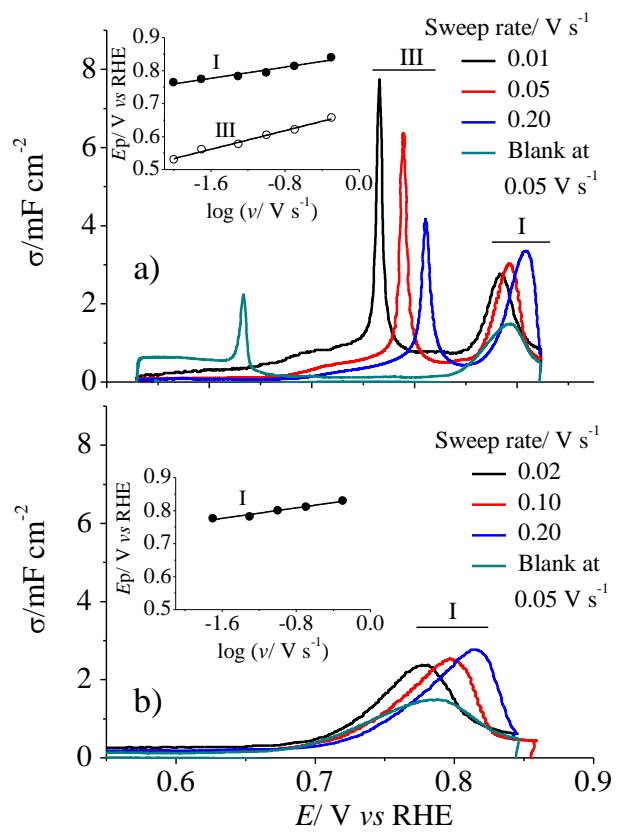

Figure 6. CO stripping voltammetry for the $\mathrm{Pt}(554)$ electrode at different sweep rates: a) full coverage; b) only step occupied. Insert: Peak potential for $\mathrm{CO}$ electrooxidation versus the logarithm of the sweep rate

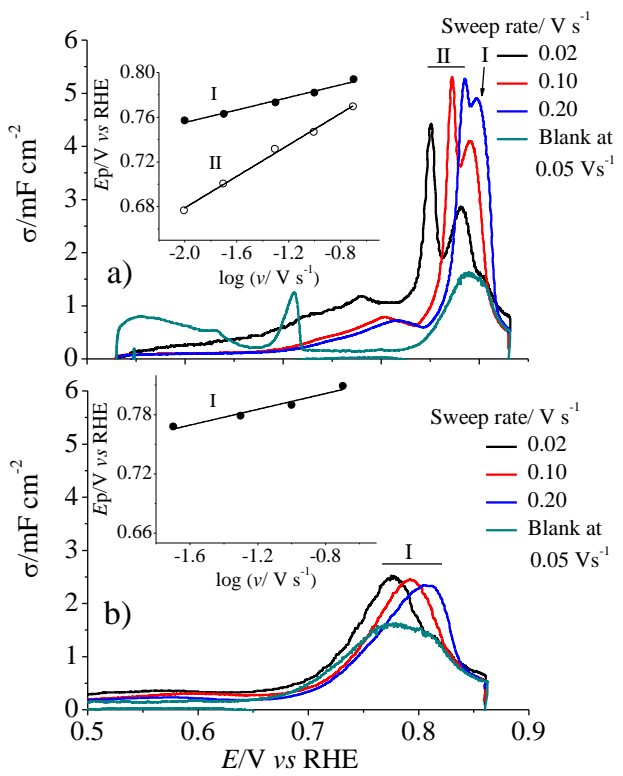

Figure 7. CO stripping voltammetry for the $\operatorname{Pt}(544)$ electrode at different sweep rates: a) full coverage; b) only step occupied. Insert: Peak potential for CO electrooxidation versus the logarithm of the sweep rate 
measurements are 40 and $120 \mathrm{mV}$ for the same processes in this potential range. ${ }^{20}$

Values of $28 \pm 2 \mathrm{mV}$ and $70 \pm 4 \mathrm{mV}$ for peak I and II respectively are obtained for the $\mathrm{Pt}(544)$ electrode at full $\mathrm{CO}$ coverage, whereas the slope for peak I in Figure $7 \mathrm{~b}$ is $40 \pm 4 \mathrm{mV}$. On the other hand, due to the difficulty in obtaining only a single type of occupied site for the $\mathrm{Pt}(875)$ electrode, the results of the effect of the scan rate in the CO stripping experiments on the $\mathrm{Pt}(875)$ electrode are only measured for full $\mathrm{CO}$ coverage (Figure 8). The values of the $\mathrm{d} E / \mathrm{d}(\log v)$ are $44 \pm 3 \mathrm{mV}, 85 \pm 3 \mathrm{mV}$ and $74 \pm 5 \mathrm{mV}$ respectively for peaks I, II and III, respectively.

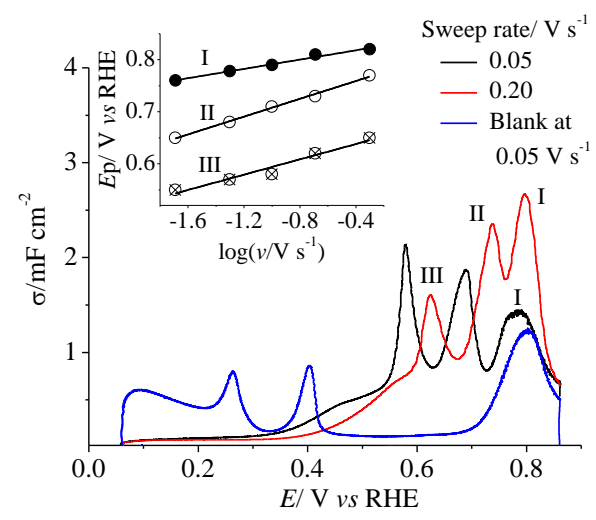

Figure 8. CO stripping voltammetry for the $\mathrm{Pt}(875)$ electrode at different sweep rate, with a $\mathrm{CO}$ full coverage. Insert: Peak potential for $\mathrm{CO}$ electrooxidation versus the logarithm of the sweep rate.

\subsection{Influence of temperature on the peak potential for electrooxidation of bonded CO} on terrace and step sites

Figures 9 and 10 report data on the influence of temperature on the voltammetric profile for $\mathrm{CO}$ stripping. As described in the above section, the experiments were done with two different $\mathrm{CO}$ coverages: in Figures 9a and 10a the $\mathrm{CO}$ stripping corresponds to fully blocked surfaces, whereas in Figures $9 \mathrm{~b}$ and $10 \mathrm{~b} \mathrm{CO}$ oxidation is done when hydrogen adsorption on the terrace is recovered by previous CO stripping. For $\operatorname{Pt}(554)$ and $\operatorname{Pt}(544)$, the peak potentials shift toward less positive values as temperature 
Published in The Journal of Physical Chemistry C. 2013, 117(6): 2903-2913. doi:10.1021/jp311499s

increases. In fact, the representation of the peak potential $v s$. $T$ is linear in the temperature range between 283 and $313 \mathrm{~K}$. The behavior is similar to that reported in acidic medium, ${ }^{27}$ although the temperature range used here is smaller. The measured 


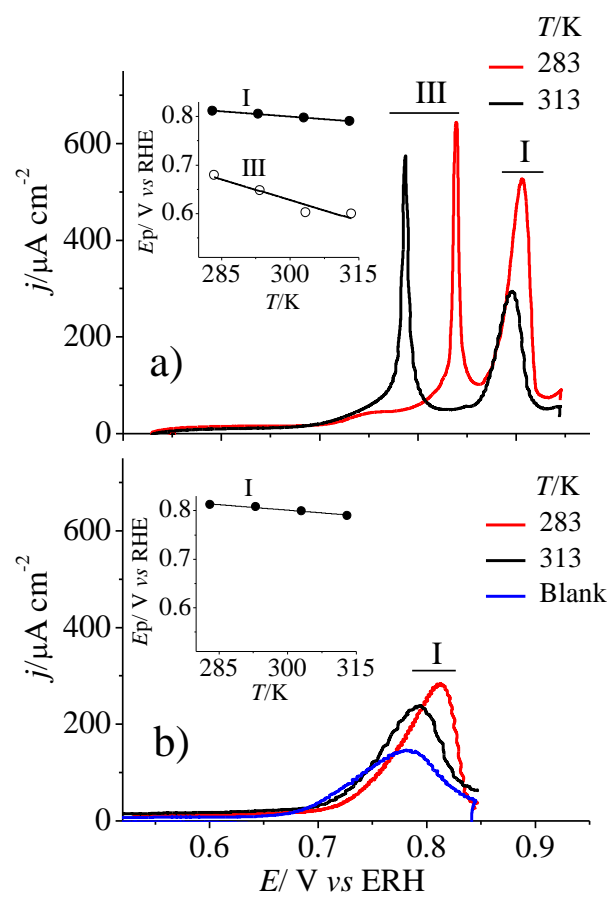

Figure 9. CO stripping voltammetry for the $\mathrm{Pt}(554)$ electrode at different temperatures: a) full coverage; b) only step occupied. Insert: Peak potential for $\mathrm{CO}$ electrooxidation versus temperature. Sweep rate: $0.10 \mathrm{~V} \mathrm{~s}^{-1}$.

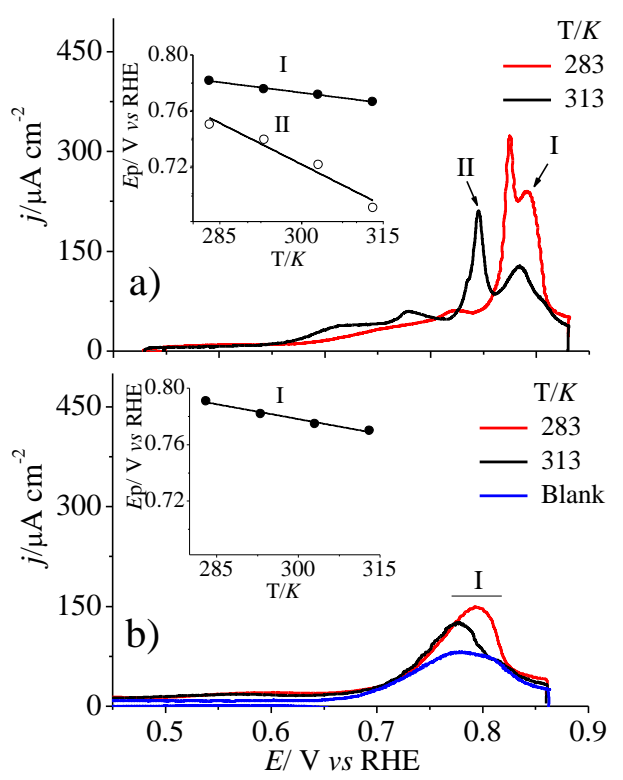

Figure 10. CO stripping voltammetry for the $\operatorname{Pt}(544)$ electrode at different temperatures: a) full coverage; b) only step occupied. Insert: Peak potential for CO electrooxidation versus temperature. Sweep rate: $0.05 \mathrm{~V} \mathrm{~s}^{-1}$

slopes in the graph are $-0.72 \pm 0.02 \mathrm{mV} \mathrm{K}^{-1}$ and $-2.9 \pm 0.13 \mathrm{mV} \mathrm{K}^{-1}$ for peak I and III, respectively, for the $\operatorname{Pt}(554)$ electrode at full coverage, whereas a value of $-0.75 \pm 0.04$ $\mathrm{mV} \mathrm{K}^{-1}$ is obtained when only the steps are covered. This means that apparent CO 
stripping activation energy (Table 1) for peak I is independent of the terrace coverage, within the error limits.

In comparison, the slope $\mathrm{d} E_{\mathrm{p}} / \mathrm{d} T$ for peak $\mathrm{I}$ is $-0.50 \pm 0.02 \mathrm{mV} \mathrm{K} \mathrm{K}^{-1}$, for the $\operatorname{Pt}(544)$ electrode, a value lower than in the previous case. It should be mentioned, however, that peak II shifts with temperature $\sim 50 \%$ less than peak III, $\mathrm{d} E_{\mathrm{p}} / \mathrm{d} T=-1.9 \pm 0.23 \mathrm{mV} \mathrm{K}^{-1}$. Interestingly, for the $\operatorname{Pt}(544)$ electrode with only the steps covered, the slope of peak I is $-0.70 \pm 0.05 \mathrm{mV} \mathrm{K}{ }^{-1}$, quite similar to the case of $\operatorname{Pt}(554)$.

The voltammetric profile of electrooxidation of $\mathrm{CO}$ at full coverage for $\operatorname{Pt}(875)$ for various temperatures is given in Figure 11 . The slopes $\mathrm{d} E_{\mathrm{p}} / \mathrm{d} T$ for peaks I, II and III are respectively $-1.25 \pm 0.08 \mathrm{mV} \mathrm{K}^{-1},-2.2 \pm 0.15 \mathrm{mV} \mathrm{K}^{-1}$ and $-2.9 \pm 0.3 \mathrm{mV} \mathrm{K}^{-1}$. It should be noted that peak I shift is larger than in the two previous cases, probably because the terrace length is shorter in the kinked surface used. In contrast, the slope for peak II only increases 20\% and peak III remains constant within experimental error. All numerical results, as well as an estimation of the activation energies ${ }^{27}$ are given in

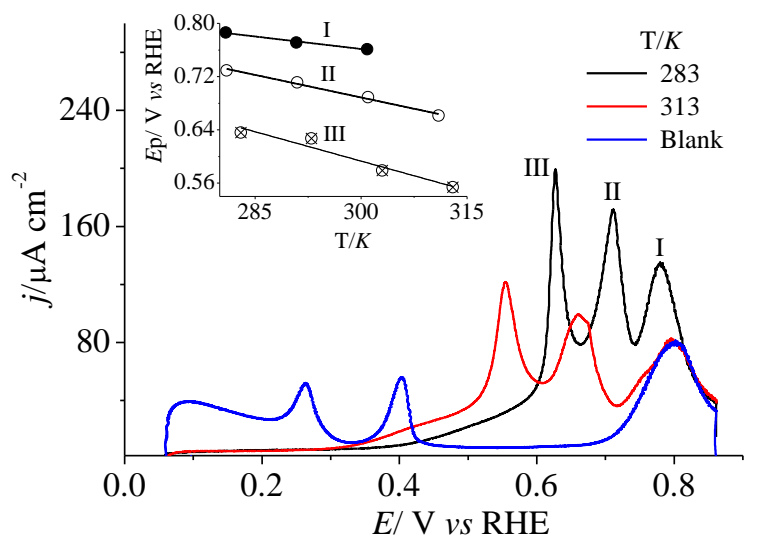

Figure 11. CO stripping voltammetry for the $\mathrm{Pt}(875)$ electrode at different temperatures, with full $\mathrm{CO}$ coverage. Insert: Peak potential for $\mathrm{CO}$ electrooxidation versus temperature. Sweep rate: $0.05 \mathrm{~V} \mathrm{~s}^{-1}$.

Table I. All data point out, however, that CO oxidation under peaks II and III are more 
temperature dependent, as compared to the oxidation of $\mathrm{CO}$ under peak I. This latter process is also independent of the local coverage on the terraces.

\subsection{On the stoichiometry of $\mathrm{CO}$ on step sites}

Figure 12 compares two CO stripping voltammetries performed with fully covered $\mathrm{CO}$ surfaces and only $\mathrm{CO}$ adsorbed on steps, which was obtained after partial CO stripping. The experiments prove the great stability of CO on (110) or (100) steps compared with CO of (111) terraces, as was observed in Figures 6 and 7 for various sweep rates. The integrated charge density under peak for hydrogen adsorption on step sites is ca. $\sim 27.2 \mu \mathrm{C} \mathrm{cm}^{-2}$ for the $\mathrm{Pt}(554)$ electrode and the corresponding charge density due to $\mathrm{CO}$ electrooxidation is $\sim 49 \mu \mathrm{C} \mathrm{cm}^{-2}$. This value has been obtained from the charge density difference between the stripping $\mathrm{CO}$ charges for both coverages. These

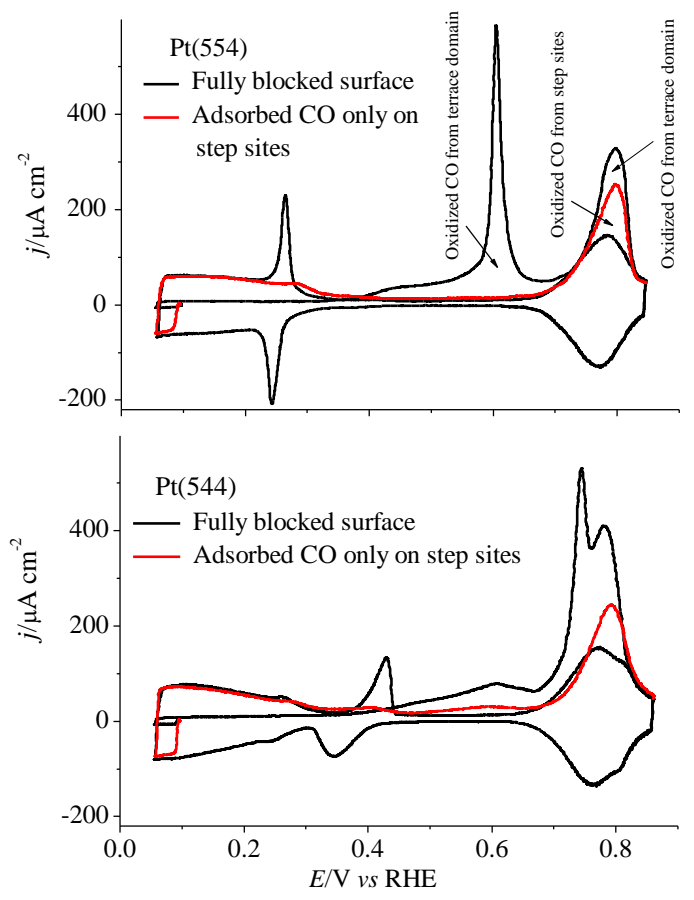

Figure 12. Comparison of two stripping voltammograms with different $\mathrm{CO}$ coverages for a) $\mathrm{Pt}(554)$ and b) $\mathrm{Pt}(544)$ electrodes. Sweep rate: $0.10 \mathrm{~V} \mathrm{~s}^{-1}$. 
results give rise to a value for the number of electrons exchanged per platinum site involved in the oxidation of peak $\mathrm{I}(n)$ equal to 1.83 . In this calculation, the $\mathrm{CO}$ stripping charge is not corrected by double layer recovery. ${ }^{37}$ It should be noted, however, that this correction, if necessary, should be small because there is not significant anion adsorption contribution in the step adsorption state in alkaline solutions and the charge under the peaks is almost the same as in acidic solution. In any case, the $n$ value suggests that CO would adsorb linearly on the (110) step, as it happens in acidic medium. ${ }^{4}, 38$ In the case of $\mathrm{Pt}(544)$, (Figure 12b) the hydrogen adsorption charge density for the step is $\sim 39 \mu \mathrm{C} \mathrm{cm}^{-2}$ and the $\mathrm{CO}$ stripping charge is $\sim 57 \mu \mathrm{C} \mathrm{cm}^{-2}$, without double layer corrections, as before. A similar analysis for Figure 12b gives a stoichiometric value $n \approx 1.46$. This is a significantly lower value than that measured for the (110) steps and it could suggest a preferential CO bridge adsorption on (100) step sites, in a similar way that is observed in acidic media. ${ }^{4}$

\subsection{In situ FTIR spectroscopic data}

In order to confirm the bonding mode of adsorbed $\mathrm{CO}$ on the step proposed from the charge measurements in the voltammetric profiles, FTIR experiments were carried out. Figure 13 displays the in situ FTIR spectra for adsorbed CO in two stepped $\operatorname{Pt}(17$ $1715) \equiv \operatorname{Pt}(\mathrm{s})-[16(111) \times(110)], \operatorname{Pt}(171515) \equiv \operatorname{Pt}(\mathrm{s})-[16(111) \times(100)]$ and a kinked $\operatorname{Pt}(531) \equiv \operatorname{Pt}(s)-[3(111) \times 1(110) \times(100)]$ surfaces. Positive bands in the spectra are associated with species present at the sampling potential in the interfacial region but not at the reference potential, whereas negative bands correspond to species present only at the reference potential. For the fully CO covered Pt(17 17 15) surface, the spectrum (top panel-black line) exhibits two positive broad bands centered at $\sim 2022 \mathrm{~cm}^{-1}$ and 1800 $\mathrm{cm}^{-1}$ attributed to the linear $\mathrm{CO}\left(\mathrm{CO}^{1}\right)$ and bridged bonded $\mathrm{CO}\left(\mathrm{CO}^{\mathrm{b}}\right)$, respectively. At 
lower frequencies, the broad band centered at $\sim 1636 \mathrm{~cm}^{-1}$ is related to the $\mathrm{O}-\mathrm{H}$ bending mode of water ${ }^{34}$ and the band at $\sim 1404 \mathrm{~cm}^{-1}$ can be assigned to carbonate formed at the reference potential due to the oxidation of $\mathrm{CO} .{ }^{39}$ When only the step sites are covered, the spectrum for adsorbed $\mathrm{CO}$ on the $\operatorname{Pt}\left(\begin{array}{lll}17 & 17 & 15\end{array}\right)$ surface (top panel - red line) only shows a single band due to the $\mathrm{CO}^{1}$ at $\sim 1976 \mathrm{~cm}^{-1}$. The absence of a measureable band at lower wave numbers associated to bridged bonded $\mathrm{CO}$ clearly indicates that main configuration of $\mathrm{CO}$ is linear $\mathrm{CO}$. Thus, the expected number of electrons transferred per platinum site in the $\mathrm{CO}$ oxidation process should be close to two, in agreement with the $n$ value of 1.83 found for (110) step in $\operatorname{Pt}(554)$ surface in the previous section.

When compared to the spectrum for fully $\mathrm{CO}$ covered surface, this band is shifted

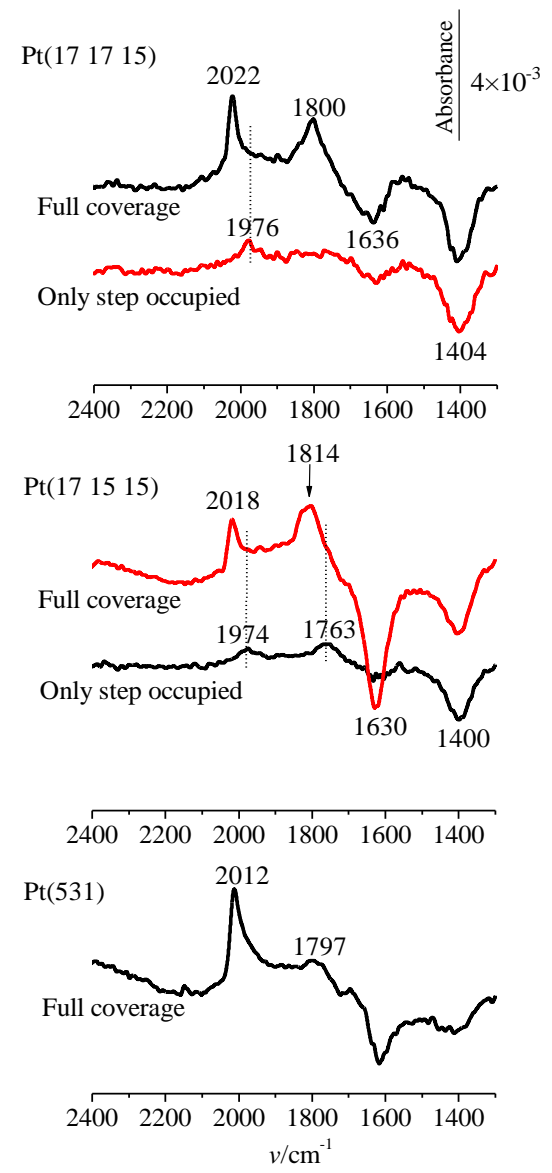

Figure 13. In situ FTIR spectra recorded at $0.20 \mathrm{~V}$ with different $\mathrm{CO}$ coverage on $\mathrm{Pt}(17,17$, 15), $\mathrm{Pt}(17,15,15)$ and $\mathrm{Pt}(531)$ single crystals. The reference potential was collected at 0.90 $\mathrm{V}$. 
ca. $46 \mathrm{~cm}^{-1}$ towards lower wavenumbers. The lower wave numbers of $\mathrm{CO}$ adsorbed on the step with respect to that measured for $\mathrm{CO}$ on the terrace clearly indicates that the energetics of $\mathrm{CO}$ bonding on the different sites is highly affected by the type of site. It should be highlighted that the band at $1976 \mathrm{~cm}^{-1}$ cannot be detected in the spectra for the fully covered surface (not even a small shoulder is present in this wave number) due to the coupling of this band with that for $\mathrm{CO}$ on the terrace. Consequently all the band intensity due to the $\mathrm{CO}^{1}$ on step sites is transferred toward the band at higher frequency $\left(\right.$ at $\left.\sim 2022 \mathrm{~cm}^{-1}\right){ }^{25}$

For fully CO covered $\operatorname{Pt}\left(\begin{array}{lll}17 & 17 & 15\end{array}\right)$ surface, the Stark effect $d v_{\mathrm{CO}} / \mathrm{d} E$ is 48 $\mathrm{cm}^{-1} \mathrm{~V}^{-1}$ and $125 \mathrm{~cm}^{-1} \mathrm{~V}^{-1}$ for $\mathrm{CO}^{1}$ and $\mathrm{CO}^{\mathrm{b}}$, respectively. When compared to the values reported on the $\mathrm{Pt}(111),\left(37 \mathrm{~cm}^{-1} \mathrm{~V}^{-1}\right.$ and $81 \mathrm{~cm}^{-1} \mathrm{~V}^{-1}$ for $\mathrm{CO}^{1}$ and $\mathrm{CO}^{\mathrm{b}}$, respectively), the values measured for the stepped surfaces are higher. ${ }^{18}$ On the other hand, for $\mathrm{CO}^{1}$ only on step sites in same surface, the Stark effect found was $126 \mathrm{~cm}^{-1} \mathrm{~V}^{-1}$. Kim and Korzeniewski ${ }^{12}$ have also reported high Stark effect for CO adsorbed on step site in controlled dosing experiments in acidic media. The nature of high Stark effect for CO on step site is unknown, but as before, it reveals that the $\mathrm{CO}$ adsorption properties are very sensitive to the surface structure.

In the case of $\operatorname{Pt}\left(\begin{array}{lll}17 & 15 & 15\end{array}\right)$, the spectrum exhibits two bands for a fully CO covered surface: one at $\sim 2018 \mathrm{~cm}^{-1}$ due to $\mathrm{CO}^{1}\left(d v_{\mathrm{CO}} / \mathrm{d} E=49 \mathrm{~cm}^{-1} \mathrm{~V}^{-1}\right)$ and another band at $1803-1827 \mathrm{~cm}^{-1}$ attributed to $\mathrm{CO}^{\mathrm{b}}\left(d v_{\mathrm{CO}} / \mathrm{d} E=86 \mathrm{~cm}^{-1} \mathrm{~V}^{-1}\right)$. The Stark effect values for this surface are comparable to those obtained for the other stepped surface. When CO is only adsorbed on the step sites (red line), the spectrum clearly shows two CO bands at $\sim 1974 \mathrm{~cm}^{-1}$ and $\sim 1763 \mathrm{~cm}^{-1}$ due to the $\mathrm{CO}^{1}$ and $\mathrm{CO}^{\mathrm{b}}$, respectively. Unlike the previous case, both $\mathrm{CO}^{1}$ and $\mathrm{CO}^{\mathrm{b}}$ bands can be observed on the spectrum for $\mathrm{CO}$ adsorbed on the step, which implies that both adsorption configurations for $\mathrm{CO}$ are observed on this type 
of site. The presence of linear and bridge bonded $\mathrm{CO}$ in similar ratio is in agreement with the stoichiometry value $n \approx 1.46$ electrons transferred per Pt step site found for the (100) step in $\mathrm{Pt}(544)$ surface. Again, due to the strong coupling between CO bands, these bands are absent from the spectrum at full coverage.

For the $\mathrm{Pt}(531)$ kinked surface, the experiment was performed only for $\mathrm{CO}$ at full coverage. Thus, the spectrum shows a high band at $2012 \mathrm{~cm}^{-1}$ atributted to the $\mathrm{CO}^{1}$ and a small band at $1797 \mathrm{~cm}^{-1}$ due to the $\mathrm{CO}^{\mathrm{b}}$. In comparison to the stepped surfaces, the $\mathrm{CO}$ on this kinked surface adsorbed essentialy with linear mode.

\section{Discussion}

It is clear that the presence of steps is modifying the adsorption/stripping of $\mathrm{CO}$ on the single crystal electrode surfaces vicinal to the $\operatorname{Pt}(111)$ surface. The presence of a step creates a perturbation that modifies the energetics of the neighboring sites, originating a surface dipole due to the Smoluchoswki effect. ${ }^{40}$ Based on this model, an ideal stepped surface can be treated as a sequence of step-dipole moments appearing where the symmetry of the terrace is broken. In this dipole, there is a flow of charge density from the upper step side towards lower step side. Thus, the upper part of the step is the positive part of the dipole whereas the lower part carries a partial negative charge. The size of the dipole depends on the symmetry and chemical substrate composition, ${ }^{41}$ and the presence of the dipole changes the local work function. ${ }^{42}$ Thus, the upper and lower parts of the step are affected in opposite directions and it is expected that the change in the energetics of adsorption processes will have the opposite sign in the two sides of the step. In the voltammetric profile of these stepped electrodes, the presence of the step gives rise to a characteristic peak in the blank solution associated with the competitive adsorption of $\mathrm{H}$ and probably OH/O. ${ }^{35}$ Although there is 
no experimental evidence on the exact adsorption position of those species in electrochemical environments, theoretical and UHV studies suggest that the adsorption of these species is taking place on the upper part of the step. ${ }^{10}$ In following, we will assume that those processes will occur on the upper part of the step, as has been done previously in similar models. ${ }^{19}$ However, if the peaks in the voltammograms are linked to the adsorption of $\mathrm{H}$ and probably $\mathrm{OH} / \mathrm{O}$ on the lower part of the step, the conclusions reached from the analysis will be equivalent to that obtained here with the exchange of the upper and lower step sites.

The direct analysis of the results presented in Figures 2-4 indicates that the initial stages of $\mathrm{CO}$ adsorption on the stepped surfaces in this medium involve preferentially the sites associated with the adsorption of $\mathrm{H}$ (and probably $\mathrm{OH}$ ) on the (110) and (100) steps. As aforementioned, we will assume that the adsorption site is located in the upper part of the step, since $\mathrm{CO}$ is blocking the adsorption of $\mathrm{H} / \mathrm{OH}$. From the charge evolution of the terrace and step sites, it is clear that the CO adsorption process is preferentially occurring on these sites, since the peaks corresponding to these processes have completely disappeared well before terrace sites have been occupied. Moreover, the preference for adsorption on the upper part of the step sites increases with the step coverage, as revealed by the fast diminution of the charge of these peaks when the CO site occupancy on this type of site is higher than $50 \%$ (Figure 3). It should be remarked that the preference for $\mathrm{CO}$ adsorption on these sites is not absolute, since $\mathrm{CO}$ is already adsorbed on the terrace sites well before the adsorption on the step site is complete. When $\mathrm{CO}$ molecules are arriving at the surface, the $\mathrm{CO}$ adsorption probability on the upper part of the step is higher than that corresponding to the rest of the sites. Thus, it can be said that the upper part of the step has higher adsorption energy for CO than the terraces, as has been proposed by DFT studies. ${ }^{10}$ It is also possible that $\mathrm{CO}$ adsorbed in 
the step modifies the adsorption energy of the neighboring step sites so that the adsorption rate on the step increases with the $\mathrm{CO}$ coverage. Additionally, the in situ FTIR experiments demonstrates that the bonding characteristics for adsorbed $\mathrm{CO}$ strongly depend on the step symmetry. In case of $\operatorname{Pt}(\mathrm{s})-[n(111) \times(110)]$ surfaces, the upper part of step is occupied essentially by linear CO; on the other hand, the upper part of the step is covered with both linear and bridge bonded $\mathrm{CO}$ for the $\mathrm{Pt}(\mathrm{s})$ $[n(111) \times(100)]$ surfaces.

These results are different from those obtained in acid media, ${ }^{14}$ where CO adsorption does not show a preference for the step sites $v s$. terrace sites for the surfaces with (110) steps. It should be borne in mind that there is ca. $0.7 \mathrm{~V}$ in potential difference in the SHE scale between $0.1 \mathrm{M} \mathrm{HClO}_{4}$ and $0.1 \mathrm{M} \mathrm{NaOH}$, which implies that the charge density on the metallic side of the electrode is significantly different. The change in absolute potential is associated with a lowering of the wave numbers for $\mathrm{CO}$ bands in $0.1 \mathrm{M} \mathrm{NaOH}$ (Figure 13) as compared with that measured in $0.1 \mathrm{M} \mathrm{HClO}_{4}{ }^{43}$. There are two possible explanations for this different behavior in the preferential site occupancy: i) there is a significant effect on the adsorption energy of $\mathrm{CO}$ due to the difference in surface charge and/or ii) diffusion rates are different in both media as has been proposed. ${ }^{21}$ In this latter case, the different diffusion rate can be also a consequence of the different charge density or electrode potential, ${ }^{44}$ which in turn affects the corrugation potential of the surface. ${ }^{45}$ It should be mentioned that a large corrugation potential generally implies a single mode for the adsorption of the molecules, which seems to be not the case of $\mathrm{CO}$. However, due to the large energy transfer between $\mathrm{CO}$ adsorption bands, it is not possible to determine the exact binding distribution of the $\mathrm{CO}$ molecules using only the spectra from the FTIR bands. ${ }^{26}$ In acid media, fast diffusion allows for a random distribution of the molecules over the surface, independently of the 
initial adsorption site, whereas slow diffusion will tend to freeze the initial configuration upon adsorption.

The site release when $\mathrm{CO}$ is slowly oxidized follows similar trends. It occurs first on the terrace sites and later on the upper part of the step sites. However, in this case, the preference for the stripping from terrace sites is absolute: the step sites are only released when the $\mathrm{CO}$ has been completely desorbed from terrace sites. A very similar model for the site released has been proposed by Koper and col. ${ }^{19-20}$ The major difference with that model is the absence of adsorbed carbonate in the model, which was used to justify the low mobility of $\mathrm{CO}$ (or low reactivity) towards the lower part of the step sites. Carbonate is not specifically adsorbed at this $\mathrm{pH} .{ }^{46}$

Unlike the adsorption process, which is initially a pure stochastic process, the release of the occupied sites, that is, the oxidation of the adsorbed $\mathrm{CO}$, requires the interaction with other molecules (activated water or adsorbed $\mathrm{OH}$ ), so that the additional oxygen atom needed to form $\mathrm{CO}_{2}$ (or carbonate) is transferred. Thus, the activity of the different surface sites can be completely different. In acidic media, it has been proposed that the steps catalyze the oxidation of adsorbed $\mathrm{CO}$ and this process would not take place on a perfect $\mathrm{Pt}(111)$ electrode ${ }^{5}$. In alkaline medium, the presence of the step affects the oxidation, since new peaks for the oxidation (peaks II and III) are observed at lower potentials. This fact indicates that new sites that are introduced with the step are more active for the oxidation of CO. However, this new type of site cannot be in the middle of the terrace because it is not observed in well-ordered $\operatorname{Pt}(111)$ electrode or in the upper part of the step, since they are the last released sites. Thus, the only possibility is that they are associated with the lower part of the step (either the row of atoms just below the step or the next row of atoms in the terrace). Thus, the initial oxidation should take place on those sites. Once the lower part of the step is free from $\mathrm{CO}$, the remaining 
CO may diffuse to those sites and react. However, because these sites are the last ones to be occupied (they have the less favorable adsorption energy for $\mathrm{CO}$ ) it is likely that the CO diffusion from middle of the terrace toward the lower part of step is hindered. Binding energy gradients which affect surface diffusion has been also proposed previously both from experimental ${ }^{47}$ and theoretical studies ${ }^{48}$ energy gradients

For alkaline solutions, it has been shown that the charge of the peak appearing at low potentials depends on the scan rate. ${ }^{17,21}$ At high scan rates, the charge density under this peak is constant and it is equivalent to that that would have been obtained for the oxidation of $\mathrm{CO}$ on the lower part of the step transferring between one and two electrons per site, depending on the step symmetry. This means that at high scan rates, the only $\mathrm{CO}$ molecules that are being oxidized are those located on the lower part of the step. At low scan rates, the charge density increases, implying that some $\mathrm{CO}$ molecules are able to diffuse and be oxidized in the lower part of the step (which is already free). For that reason, the slow $\mathrm{CO}$ removal experiments performed in Figure 5 progressively strip $\mathrm{CO}$ from the terrace sites. On the other hand, the $\mathrm{CO}$ molecules which are adsorbed on the upper part of the step are trapped in this position due to the high adsorption in energy on this site. Thus, these molecules cannot be oxidized in the catalytic sites and, the oxidation potential is higher. Accidentally, the potential at which this $\mathrm{CO}$ is oxidized in the voltammetry coincides with that measured for the $\mathrm{CO}$ oxidation on a well ordered $\operatorname{Pt}(111)$ electrode. This model for the oxidation of $\mathrm{CO}$ is also in agreement with the results reported in Figure 4, in which peaks II and III are only observed when the surface is saturated with CO. The sites in lower part of the step are the last sites to be occupied and for that reason, the oxidation peak associated with these sites only appears at full saturation. 
There is also an additional difference between the $\mathrm{CO}$ adsorption and stripping processes. In the case of the slow adsorption experiments, the peak for $\mathrm{H} / \mathrm{OH}$ adsorption on the step does not shift in potential when the step is partially covered by $\mathrm{CO}$, whereas a measurable shift towards positive potentials is observed when similar partial $\mathrm{CO}$ coverages in the step are obtained from stripping experiments. The differences indicate that the exact distribution of the $\mathrm{CO}$ molecules on the step is different in the two situations and that different interactions between $\mathrm{H}$ and probably $\mathrm{OH}$ and $\mathrm{CO}$ exist in each case. For the slow stripping experiments, $\mathrm{CO}$ adsorption on the step affects the energetics of $\mathrm{H} / \mathrm{OH}$, and the opposite behavior is observed for the slow dosing experiments. Although the exact origin of such potential shift is not clear, the nonequivalence of the two situations clearly suggests that $\mathrm{CO}$ diffusion over the surface is slow. Even though metastable adlayers may survive in situations where diffusion is fast, the fast surface diffusion normally would imply reaching equilibrium conditions, so that identical results for a given surface coverage would have been obtained independently on how this coverage is obtained.

The dual oxidation mechanism, in which two different type of sites contribute to the oxidation of $\mathrm{CO}$ on the stepped surfaces is corroborated by the different Tafel slopes and activation energies. The $\mathrm{CO}$ oxidation process, which is linked to the lower part of the step (peaks II and III), has a Tafel slope value which is significantly different to that measured for peak I. Frequently, changes in the Tafel slope are analyzed in terms of a change in the mechanism. However, it has been shown that a change in the adsorption isotherm of $\mathrm{OH}$, which is required to oxidize $\mathrm{CO}$ in a Langmuir-Hinselwood mechanism, significantly modifies the values of the Tafel slope ${ }^{49}$ For this medium, the most probable cause for the change in the Tafel slope is the change in the isotherm for $\mathrm{OH}$ adsorption on the different sites. The same conclusion can be reached from the 
different activation energies observed for the different peaks. Additionally, a small dependency of the activation energy on total $\mathrm{CO}$ coverage demonstrates that these processes are specific for each domain of the surface.

The two stepped and the kink surfaces employed in this study indicates that the behavior of the step and kinks sites for the $\mathrm{CO}$ adsorption and stripping process is qualitatively the same. The only significant difference between the sites with (110) and (100) symmetry is the potential at which the initial oxidation of CO on these surfaces is occurring, but it does not object that the mechanism ruling these processes is the same. The only quantitative difference in this case is the energetics of the site. It is known that the dipole generated by the presence of a step depends on its symmetry. Thus, large dipoles are measured for the (110) step in comparison with the (100) step on the (111) terraces. ${ }^{50-51}$ This difference affects any adsorption process on these sites and therefore, the stripping of $\mathrm{CO}$ depends on these values.

\section{Conclusions}

In this paper, we have shown for the first time that in alkaline medium:

1. The $\mathrm{CO}$ adsorption rate onto stepped and kinked platinum surface is site dependent, and the sequence of site blockage is: (100) and (110) step sites; and finally (111) terrace sites.

2. The origin of peak multiplicity in $\mathrm{CO}$ stripping voltammetry is connected with progress of CO site occupancy.

3. The $\mathrm{CO}$ electrooxidation takes place in preferential sites and the sequence of site release is: (111) terrace sites, (110) and (100) step sites. The Tafel plots for CO oxidized in (111) terraces sites onto $\operatorname{Pt}(554)$ and $\operatorname{Pt}(544)$ are very similar, suggesting that the (111) terrace sites are the first type of sites to be released during the $\mathrm{CO}$ adlayer 
oxidation. It has been proposed that the first sites to be released upon oxidation are those close to the lower part of the step, and that the oxidation continues over the terrace. The last sites to be released are those in the upper side of the step.

4. In absence of $\mathrm{CO}$ on terrace sites, it was possible to identify by in situ FTIR spectroscopy, the adsorption configuration for CO on the step. $\mathrm{CO}$ molecules on (110) step sites are mainly linearly adsorbed whereas linear and bridged bonded $\mathrm{CO}$ can be observed on the (100) step sites.

5. The activation energy for $\mathrm{CO}$ electrooxidation is dependent whether $\mathrm{CO}$ molecules are adsorbed in step or terrace sites, and activation energy for $\mathrm{CO}$ electrooxidation on terrace sites is higher than activation energy for that reaction on step sites.

Acknowledgements: Farias, M.J.S. would like to thanks CNPq, Brazil, for financial support for his postdoctoral stay at Universidad de Alicante. This work has been financially supported by the MICINN (Spain) (project CTQ2010-16271) and Generalitat Valenciana (project PROMETEO/2009/045, -FEDER).

\section{References}

1. Lai, S. C. S.; Lebedeva, N. P.; Housmans, T. H. M.; Koper, M. T. M., Mechanisms of Carbon Monoxide and Methanol Oxidation at Single-Crystal Electrodes. Top. Catal. 2007, 46, 320-333.

2. Inkaew, P.; Zhou, W.; Korzeniewski, C., CO Monolayer Oxidation at $\operatorname{Pt}\left(\begin{array}{lll}1 & 0 & 0\end{array}\right)$ Probed by Potential Step Measurements in Comparison to $\operatorname{Pt}\left(\begin{array}{lll}1 & 1 & 1\end{array}\right)$ and Pt Nanoparticle Catalyst. J. Electroanal. Chem. 2008, 614, 93-100.

3. Vidal-Iglesias, F. J.; Solla-Gullón, J.; Campina, J. M.; Herrero, E.; Aldaz, A.; Feliu, J. M., CO Monolayer Oxidation on Stepped Pt(S) (N-1)(100) X (110) Surfaces. Electrochim. Acta 2009, 54, 4459-4466.

4. Chen, Q. S.; Berna, A.; Climent, V.; Sun, S. G.; Feliu, J. M., Specific Reactivity of Step Sites Towards CO Adsorption and Oxidation on Platinum Single Crystals Vicinal to Pt(111). Phys. Chem. Chem. Phys. 2010, 12, 11407-11416. 
5. Lebedeva, N. P.; Koper, M. T. M.; Feliu, J. M.; van Santen, R. A., Role of Crystalline Defects in Electrocatalysis: Mechanism and Kinetics of $\mathrm{CO}$ Adlayer Oxidation on Stepped Platinum Electrodes. J. Phys. Chem. B 2002, 106, 12938-12947.

6. Hayden, B. E.; Kretzschmar, K.; Bradshaw, A. M.; Greenler, R. G., An Infrared Study of the Adsorption of CO on a Stepped Platinum Surface. Surf. Sci. 1985, 149, 394-406.

7. Luo, J. S.; Tobin, R. G.; Lambert, D. K.; Fisher, G. B.; DiMaggio, C. L., CO Adsorption Site Occupation on $\mathrm{Pt}(335)$ : A Quantitative Investigation Using Tpd and Eels. Surf. Sci. 1992, 274, 53-62.

8. Xu, J.; Yates, J. J. T., Catalytic Oxidation of CO on Pt(335): A Study of the Active Site. J. Chem. Phys. 1993, 99, 725-732.

9. Yates, J. T., Jr., Surface Chemistry at Metallic Step Defect Sites. J. Vac. Sci. Technol. A 1995, 13, 1359-1367.

10. Hammer, B.; Nielsen, O. H.; Norskov, J. K., Structure Sensitivity in Adsorption: CO Interaction with Stepped and Reconstructed Pt Surfaces. Catal. Lett. 1997, 46, 3135 .

11. Kim, S. C.; Korzeniewski, C.; Tornquist, W. J., Site Specific Coa-Adsorption at $\mathrm{Pt}(335)$ as Probed by Ir Spectroscopy : Structural Alterations in the CO Adlayer under Aqueous Electrochemical Conditions. J. Chem. Phys. 1994, 100, 628.

12. Kim, C. S.; Korzeniewski, C., Vibrational Coupling as a Probe of Adsorption at Different Structural Sites on a Stepped Single-Crystal Electrode. Anal. Chem. 1997, 69, 2349-2353.

13. Baltruschat, H.; Bußar, R.; Ernst, S.; Hernandez, F., From Stepped Single Crystal Surfaces to Ordered Bimetallic Electrodes: Adsorption and Electrocatalysis as Studied by Dems and Stm. In In-Situ Spectroscopic Studies of Adsorption at the Electrode and Electrocatalysis, Sun, S.-G.; Christensen, P. A.; Wieckowski, A., Eds. Elsevier Science B.V.: Amsterdam, 2007; pp 471-537.

14. Farias, M. J. S.; Tanaka, A. A.; Tremiliosi, G.; Feliu, J. M., On the Apparent Lack of Preferential Site Occupancy and Electrooxidation of CO Adsorbed at Low Coverage onto Stepped Platinum Surfaces. Electrochem. Commun. 2011, 13, 338-341.

15. Love, B.; Lipkowski, J., Effect of Surface Crystallography on Electrocatalytic Oxidation of Carbon-Monoxide on Platinum-Electrodes. ACS Symp. Ser. 1988, 378, 484-496.

16. Santos, E.; Leiva, E. P. M.; Vielstich, W., CO Adsorbate on Pt(111) SingleCrystal Surfaces. Electrochim. Acta 1991, 36, 555-561.

17. Garcia, G.; Koper, M. T. M., Stripping Voltammetry of Carbon Monoxide Oxidation on Stepped Platinum Single-Crystal Electrodes in Alkaline Solution. Phys. Chem. Chem. Phys. 2008, 10, 3802-3811.

18. Garcia, G.; Rodriguez, P.; Rosca, V.; Koper, M. T. M., Fourier Transform Infrared Spectroscopy Study of CO Electro-Oxidation on $\mathrm{Pt}(111)$ in Alkaline Media. Langmuir 2009, 25, 13661-13666.

19. Garcia, G.; Koper, M. T. M., Dual Reactivity of Step-Bound Carbon Monoxide During Oxidation on a Stepped Platinum Electrode in Alkaline Media. J. Am. Chem. Soc. 2009, 131, 5384-5385.

20. Garcia, G.; Koper, M. T. M., Mechanism of Electro-Oxidation of Carbon Monoxide on Stepped Platinum Electrodes in Alkaline Media: A Chronoamperometric and Kinetic Modeling Study. Phys. Chem. Chem. Phys. 2009, 11, 11437-11446.

21. Herrero, E.; Chen, Q.-S.; Hernandez, J.; Sun, S.-G.; Feliu, J. M., Effects of the Surface Mobility on the Oxidation of Adsorbed CO on Platinum Electrodes in Alkaline 
Media. The Role of the Adlayer and Surface Defects. Phys. Chem. Chem. Phys. 2011, 13, 16762-16771.

22. Markovic, N. M.; Lucas, C. A.; Rodes, A.; Stamenkovic, V.; Ross, P. N., Surface Electrochemistry of CO on Pt(111): Anion Effects. Surf. Sci. 2002, 499, L149L158.

23. Spendelow, J. S.; Goodpaster, J. D.; Kenis, P. J. A.; Wieckowski, A., Mechanism of CO Oxidation on Pt(111) in Alkaline Media. J. Phys. Chem. B 2006, $110,9545-9555$.

24. Moskovits, M.; Hülse, J. E., Frequency Shifts in the Spectra of Molecules Adsorbed on Metals, with Emphasis on the Infrared Spectrum of Adsorbed Co. Surf. Sci. 1978, 78, 397-418.

25. Villegas, I.; Weaver, M. J., Carbon-Monoxide Adlayer Structures on Platinum(111) Electrodes - a Synergy between in-Situ Scanning-Tunneling-Microscopy and Infrared-Spectroscopy. J. Chem. Phys. 1994, 101, 1648-1660.

26. Severson, M. W.; Stuhlmann, C.; Villegas, I.; Weaver, M. J., Dipole-Dipole Coupling Effects Upon Infrared-Spectroscopy of Compressed Electrochemical Adlayers - Application to the Pt(111)/CO System. J. Chem. Phys. 1995, 103, 9832-9843.

27. Herrero, E.; Feliu, J. M.; Blais, S.; Radovic-Hrapovic, Z.; Jerkiewicz, G., Temperature Dependence of CO Chemisorption and Its Oxidative Desorption on the Pt(111) Electrode. Langmuir 2000, 16, 4779-4783.

28. Herrero, E.; Alvarez, B.; Feliu, J. M.; Blais, S.; Radovic-Hrapovic, Z.; Jerkiewicz, G., Temperature Dependence of the Coads Oxidation Process on $\operatorname{Pt}(111)$ $\mathrm{Pt}(100)$, and $\mathrm{Pt}(110)$ Electrodes. J. Electroanal. Chem. 2004, 567, 139-149.

29. Rincón, A.; Pérez, M. C.; Cuesta, A.; Gutiérrez, C., Dependence on the CO Admission Potential of the Activation Energy of the Electrooxidation of Adsorbed CO on Pt. Electrochem. Commun. 2005, 7, 1027-1032.

30. Climent, V.; Garcia-Araez, N.; Herrero, E.; Feliu, J., Potential of Zero Total Charge of Platinum Single Crystals: A Local Approach to Stepped Surfaces Vicinal to Pt(111). Russ. J. Electrochem. 2006, 42, 1145-1160.

31. Herrero, E.; Climent, V.; Feliu, J. M., On the Different Adsorption Behavior of Bismuth, Sulfur, Selenium and Tellurium on a Pt(775) Stepped Surface. Electrochem. Commun. 2000, 2, 636-640.

32. Clavilier, J.; Armand, D.; Sun, S. G.; Petit, M., Electrochemical Adsorption Behaviour of Platinum Stepped Surfaces in Sulphuric Acid Solutions J. Electroanal. Chem. 1986, 205, 267-277.

33. Herrero, E.; Orts, J. M.; Aldaz, A.; Feliu, J. M., Scanning Tunneling Microscopy and Electrochemical Study of the Surface Structure of $\operatorname{Pt}(10,10,9)$ and $\operatorname{Pt}(11,10,10)$ Electrodes Prepared under Different Cooling Conditions. Surf. Sci. 1999, 440, 259-270.

34. Iwasita, T.; Nart, F. C., In Situ Infrared Spectroscopy at Electrochemical Interfaces. Prog. Surf. Sci. 1997, 55, 271-340.

35. van der Niet, M. J. T. C.; Garcia-Araez, N.; Hernández, J.; Feliu, J. M.; Koper, M. T. M., Water Dissociation on Well-Defined Platinum Surfaces: The Electrochemical Perspective. Catal. Today 2013, 202, 105-113.

36. Laviron, E., General Expression of the Linear Potential Sweep Voltammogram in the Case of Diffusionless Electrochemical Systems. J. Electroanal. Chem. 1979, 101, 19-28.

37. Cuesta, A.; Perez, M. D. P.; Rincon, A.; Gutierrez, C., Adsorption Isotherm of CO on Pt(111) Electrodes. ChemPhysChem 2006, 7, 2346-2351. 
38. Pastor, E.; Rodriguez, J. L.; Iwasita, T., A Spectroscopic Proof of a Surface Equilibrium between on Top and Bridge Bonded $\mathrm{CO}$ at $\mathrm{Pt}(110)$ in Acid Solution. Electrochem. Commun. 2002, 4, 959-962.

39. Iwasita, T.; Rodes, A.; Pastor, E., Vibrational Spectroscopy of Carbonate Adsorbed on $\mathrm{Pt}(111)$ and $\mathrm{Pt}(110)$ Single-Crystal Electrodes. J. Electroanal. Chem. 1995, 383, 181-189.

40. Smoluchowski, R., Anisotropy of the Electronic Work Function of Metals. Physical Review 1941, 60, 661-674.

41. Fawcett, W. R., The Ionic Work Function and Its Role in Estimating Absolute Electrode Potentials. Langmuir 2008, 24, 9868-9875.

42. Jia, J. F.; Inoue, K.; Hasegawa, Y.; Yang, W. S.; Sakurai, T., Variation of the Local Work Function at Steps on Metal Surfaces Studied with Stm. Phys. Rev. B 1998, $58,1193-1196$.

43. Rodes, A.; Gómez, R.; Feliu, J. M.; Weaver, M. J., Sensitivity of Compressed Carbon Monoxide Adlayers on Platinum(Iii) Electrodes to Long-Range Substrate Structure: Influence-of Monoatomic Steps. Langmuir 2000, 16, 811-816.

44. Giesen, M., Step and Island Dynamics at Solid/Vacuum and Solid/Liquid Interfaces. Prog. Surf. Sci. 2001, 68, 1-153.

45. Cometto, F. P.; Paredes-Olivera, P.; Macagno, V. A.; Patrito, E. M., Density Functional Theory Study of the Adsorption of Alkanethiols on $\mathrm{Cu}(111), \operatorname{Ag}(111)$, and $\mathrm{Au}(111)$ in the Low and High Coverage Regimes. J. Phys. Chem. B 2005, 109, 2173721748.

46. Morallon, E.; Vazquez, J. L.; Aldaz, A., Electrochemical-Behavior of Basal Single-Crystal Pt Electrodes in Alkaline-Medium. J. Electroanal. Chem. 1990, 288, 217-228.

47. Massong, H.; Wang, H.; Samjeské, G.; Baltruschat, H., The Co-Catalytic Effect of Sn, Ru and Mo Decorating Steps of Pt(111) Vicinal Electrode Surfaces on the Oxidation of Co. Electrochim. Acta 2001, 46, 701-707.

48. Koper, M. T. M.; Lebedeva, N. P.; Hermse, C. G. M., Dynamics of CO at the Solid/Liquid Interface Studied by Modeling and Simulation of CO Electro-Oxidation on Pt and Ptru Electrodes. Faraday Discuss. 2002, 121, 301-311.

49. Angelucci, C. A.; Herrero, E.; Feliu, J. M., Modeling CO Oxidation on $\operatorname{Pt}(111)$ Electrodes. J. Phys. Chem. C 2010, 114, 14154-14163.

50. Besocke, K. B.; Krahl-Urban, B.; Wagner, H., Dipole Moments Associated with Edge Atoms: A Comparative Study on Stepped Pt, Au and W Surfaces. Surf. Sci. 1977, 68, 39-46.

51. Gómez, R.; Climent, V.; Feliu, J. M.; Weaver, M. J., Dependence of the Potential of Zero Charge of Stepped Platinum (111) Electrodes on the Oriented StepEdge Density: Electrochemical Implications and Comparison with Work Function Behavior. J. Phys. Chem. B 2000, 104, 597-605. 
Table 1. Activation energy $\left(E_{\mathrm{a}} / \mathrm{kJ} \mathrm{mol}^{-1}\right)$ obtained for the $\mathrm{CO}$ oxidation with different sites on surface

\begin{tabular}{|c|c|c|c|c|}
\hline \multicolumn{4}{|c|}{ For saturated $\mathrm{CO}$ coverage } & \multirow{2}{*}{$\begin{array}{l}\text { For CO only on } \\
\text { step sites } \\
\text { Peak I } \\
E_{\mathrm{a}} / \mathrm{kJ} \mathrm{mol}^{-1}\end{array}$} \\
\hline & $\begin{array}{l}\text { Peak I } \\
\mathrm{E}_{\mathrm{a}} / \mathrm{kJ} \mathrm{mol}^{-1}\end{array}$ & $\begin{array}{l}\text { Peak II } \\
E_{\mathrm{a}} / \mathrm{kJ} \mathrm{mol}^{-1}\end{array}$ & $\begin{array}{l}\text { Peak III } \\
E_{\mathrm{a}} / \mathrm{kJ} \mathrm{mol}^{-1}\end{array}$ & \\
\hline$\overline{P t}(554)$ & $97 \pm 5$ & & $144 \pm 12$ & $98 \pm 3$ \\
\hline $\operatorname{Pt}(544)$ & $88 \pm 2$ & $126 \pm 8$ & & $94 \pm 3$ \\
\hline $\operatorname{Pt}(875)$ & $105 \pm 4$ & $132 \pm 5$ & $142 \pm 10$ & \\
\hline$\overline{P t}(111)^{\mathrm{a}}$ & $135-139$ & & & \\
\hline
\end{tabular}


TOC

CO adsorption order

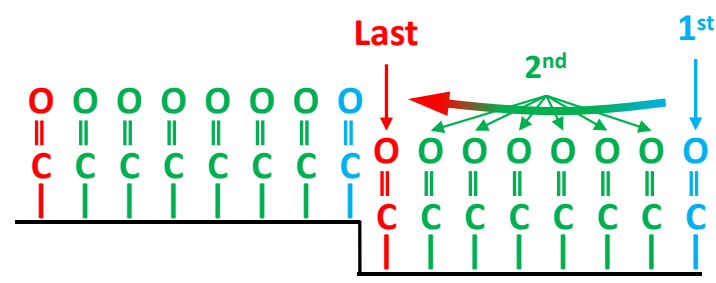

CO oxidation order

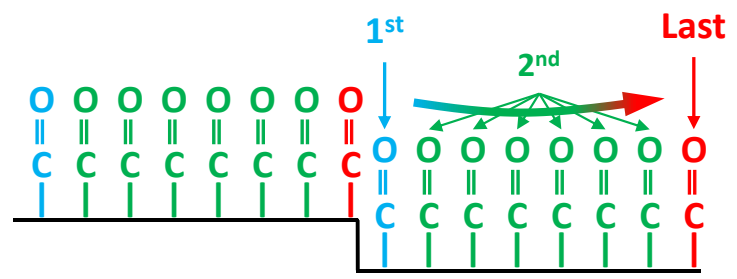

Pt(111) vicinal surfaces in alkaline media 